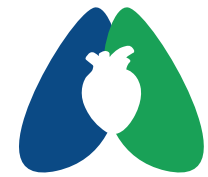

ASSOBRAFIR C I Ê N C I A

\section{Características clínicas de pacientes atendidos pela equipe de fisioterapia na enfermaria de um hospital de pequeno porte na região Litoral Norte do Rio Grande do Sul}

\author{
Clinical characteristics of patients attended by the physiotherapy \\ team in the ward of a small hospital in the North Coast of Rio \\ Grande do Sul
}

Tainara da Silva Lopes da Silva' (1); Tais da Silva Silveira' (1);

Cibele Castilho da Silveira' (1); Marjorie da Silva Rafael ${ }^{1}$ (1);

Cristiane Helena de Oliveira Sena² (1); João Paulo Heinzmann-Filho1* (1)

\section{Resumo}

Introdução: a hospitalização envolve o manejo e o tratamento de várias condições clínicas. O fisioterapeuta precisa conhecer as características dessas enfermidades para melhor atender e direcionar os recursos terapêuticos no ambiente hospitalar. Objetivo: descrever as características clínicas de pacientes atendidos pela equipe de fisioterapia na enfermaria de um hospital de pequeno porte. Métodos: trata-se de um estudo observacional, do tipo retrospectivo. Foram selecionados prontuários de pacientes ( $>18$ anos) atendidos pela equipe de fisioterapia na enfermaria de um hospital de pequeno porte no município de Osório-RS. Em contrapartida, excluíram-se aquelas fichas sem a identificação dos sujeitos e o motivo de internação reportado. Os dados foram oriundos de agosto de 2018 até dezembro de 2019. Para fins estatísticos, utilizou-se apenas análise descritiva. Resultados: os prontuários totalizaram 167 pacientes (idade: 69,9 anos), com predomínio de idosos e do sexo feminino. As doenças pulmonares foram os principais motivos de hospitalização (35,3\%), incluindo a pneumonia, a doença pulmonar obstrutiva crônica [DPOC] e o derrame pleural. As medicações (princípio ativo) prevalentes foram os anti-inflamatórios não esteroides (86,8\%), os analgésicos (49,1\%) e os antibióticos (46,7\%). A mediana de hospitalização foi de 5,0 dias, sendo que $15,9 \%$ dos pacientes necessitaram de suporte nutricional e $63,9 \%$ deles utilizaram algum dispositivo de oxigenoterapia. O escore de mobilidade foi de 15,0 pontos. Conclusão: os achados do estudo evidenciaram predomínio de mulheres, da faixa etária idosa e de pouco comprometimento clínico. As doenças pulmonares, como a pneumonia e a DPOC, foram os principais motivos de hospitalização dos pacientes atendidos pela fisioterapia.

${ }^{1}$ Centro Universitário Cenecista de Osório, Osório, RS, Brasil ${ }^{2}$ Hospital São Vicente de Paulo, Osório, RS, Brasil

Palavras-chave: Paciente; Perfil Epidemiológico; Hospitalização; Fisioterapia.

\begin{abstract}
Introduction: hospitalization involves the management and treatment of various clinical conditions. The physiotherapist needs to know the characteristics of these diseases in order to better attend and direct the therapeutic resources in the hospital environment. Objective: to describe the clinical characteristics of patients treated by the physiotherapy team in the ward of a small hospital. Methods: This is an observational, retrospective study. Medical records of patients (> 18 years) attended by the physiotherapy team in the ward of a small hospital in the municipality of Osório-RS were selected. On the other hand, those forms without the identification of the subjects and the reason for hospitalization were excluded. The data came from August 2018 until December 2019. For statistical purposes, only descriptive analysis was used. Results: the medical records totaled 167 patients (age: 69.9 years), with a predominance of elderly and female. Lung diseases were the main reasons for hospitalization (35.3\%), including pneumonia, chronic obstructive pulmonary disease [COPD] and pleural effusion. The prevalent medications (active ingredient) were non-steroidal anti-inflammatory drugs (86.8\%), analgesics (49.1\%) and antibiotics (46.7\%). The median hospital stay was 5.0 days, with $15.9 \%$ of the patients needing nutritional support and $63.9 \%$ of them using an oxygen therapy device. The mobility score was 15.0 points. Conclusion: the findings of the study showed a predominance of women, of the elderly age group and of little clinical impairment. Lung diseases, such as pneumonia and COPD, were the main reasons for hospitalization of patients treated by physiotherapy.
\end{abstract}

Keywords: Patient; Epidemiological Profile; Hospitalization; Physiotherapy. 


\section{INTRODUÇÃO}

A hospitalização pode ser entendida como a admissão ou a permanência em um estabelecimento hospitalar, tanto público como privado, que esteja equipado com todos os recursos necessários para o tratamento dos doentes ${ }^{1,2}$.

Dados apontam que, independente do sexo, a proporção de indivíduos que necessitam desse amparo terapêutico é semelhante. No entanto, existe predomínio de hospitalização nas faixas etárias extremas. Nas crianças, as principais causas são as doenças do aparelho respiratório, infeccioso e parasitário. Já nos idosos, destacam-se a doença pulmonar obstrutiva crônica (DPOC), o acidente vascular encefálico (AVE), a hipertensão arterial sistêmica (HAS) e as neoplasias ${ }^{3-5}$.

Neste sentido, os profissionais envolvidos no tratamento de enfermos no ambiente hospitalar necessitam de amplo conhecimento e manejo terapêutico, já que prestam assistência para várias patologias e condições clínicas. O fisioterapeuta, neste contexto, objetiva otimizar a funcionalidade, trabalhando sobre os aspectos cardiorrespiratórios e musculoesqueléticos ${ }^{6-9}$. Atua na profilaxia e no tratamento de infecção pulmonar, trombose venosa profunda (TVP), lesão por pressão, além de auxiliar na manutenção do trofismo muscular. Também manuseia dispositivos de oxigenoterapia e ventilação mecanica ${ }^{9-12}$.

Evidências científicas apontam que a inserção do fisioterapeuta contribui para redução do tempo de hospitalização, menor frequência de hiperoxia na enfermaria e na Unidade de Terapia Intensiva (UTI), sucesso no desmame/extubação e fornece um olhar diferenciado para o suporte ventilatório ${ }^{8,13}$. Além disso, parece que a fisioterapia impacta positivamente sobre alguns desfechos psicossociais e fatores interligados a qualidade de vida ${ }^{14}$.

Apesar dos resultados favoráveis quanto à inserção do fisioterapeuta neste contexto, a literatura científica ainda é restrita no que se refere à descrição das características clínicas de pacientes atendidos por estes profissionais no ambiente hospitalar 5,13,15-17. Um destes estudos ${ }^{5}$ foi realizado em Pelotas-RS e reportou predomínio de homens, sendo a HAS e a diabetes mellitus (DM), as patologias mais frequentes. Já outra pesquisa conduzida em Santa Maria-RS, constatou que a pneumonia foi a principal causa de hospitalização ${ }^{17}$. Entretanto, estes estudos são limitados quanto ao setor, à faixa etária e as informações clínicas reportadas nos trabalhos, o que impossibilita a análise minuciosa destes resultados. Além do mais, nenhum estudo foi desenvolvido na região Litoral Norte do Rio Grande do Sul11,15,17,18.

Estudos descritivos e epidemiológicos visam contribuir para futuras campanhas de prevenção e também estimular e reconstruir medidas de saúde pública, dentro das peculiaridades de cada comunidade ${ }^{19}$. Também auxiliam no direcionamento de recursos voltados a educação continuada para os profissionais do próprio local. Assim, o objetivo do estudo foi descrever as características clínicas de pacientes atendidos pela equipe de fisioterapia na enfermaria de um hospital de pequeno porte.

\section{MÉTODOS}

Trata-se de um estudo observacional, do tipo retrospectivo. Foram selecionados prontuários de pacientes (>18 anos) atendidos pela equipe de fisioterapia na enfermaria, do Hospital São Vicente de Paulo (HSVP), no município de Osório-RS. Em contrapartida, excluíramse aquelas fichas sem a identificação dos sujeitos (nome, sexo, idade e o motivo de internação reportado). Os dados foram extraídos de prontuários do período de agosto de 2018 até dezembro de 2019.

A extração dos dados foi realizada por duas pesquisadoras e revisado pelo pesquisador responsável do estudo. Esse processo funcionou a partir da extração das informações contidas nas fichas de avaliação para posterior inserção em uma planilha do Microsoft Excel®. Os dados foram revisados para evitar possíveis vieses na mensuração dos dados.

Foram extraídos os seguintes dados: data de internação, data de avaliação fisioterapêutica, dias de hospitalização, motivo de internação, idade, sexo, doenças associadas, princípio ativo do fármaco em uso, acesso venoso, suporte nutricional e ventilatório, necessidade de aspiração de vias aéreas superiores (VAS), restrição ao leito e escore de mobilidade ${ }^{20}$.

O motivo de internação foi classificado em sete grupos para facilitar a apresentação dos dados. Estes foram classificados da seguinte forma: 1) doenças pulmonares (DPOC, pneumonia e derrame pleural); 2) doenças cérebro/ cardiovasculares (AVE, insuficiência cardíaca congestiva [ICC], HAS e TVP); 3) doenças renais (insuficiência renal aguda e crônica); 4) doenças neuropsicológicas (epilepsia, alzheimer e parkison); 5) doenças gastroesofágicas (refluxo gastroesofágico, diverticulite, gastrite e doença de crohn); 6) doenças metabólicas (desnutrição, síndrome metabólica e diabete mellitus); 7) doenças oncológicas (tipos de câncer). Aqueles pacientes que não apresentaram o diagnóstico médico estabelecido no momento da avaliação foram classificados como em investigação.

O escore de mobilidade foi avaliado através da escala de Perme Intensive Care Unit Mobility Score e ICU Mobility Scale $^{20}$. Esta ferramenta avalia o estado mental, potenciais barreiras à mobilidade, força funcional, mobilidade ao leito, transferências, dispositivos de auxílio para deambulação e medidas de resistência. A escala oscila entre 0 e 32 pontos, sendo que resultados maiores indicam melhor mobilidade ${ }^{20}$.

O presente estudo foi aprovado pelo Comitê de Ética em Pesquisa (CEP) do Centro Universitário Cenecista de Osório (UNICNEC) pelo CAEEE 29951520.8.0000.5591. Não foi necessário obter Termo de Consentimento Livre e 
Esclarecido (TCLE) pelo fato de que neste trabalho utilizouse apenas dados secundários. Esta pesquisa obedece à resolução do Conselho Nacional de Saúde (CNS), através das resoluções n 196/1996 e n 446/2012.

O cálculo amostral foi baseado no número médio de pacientes encaminhados para a fisioterapia nesse período no HSVP. Assim, considerando o número mínimo de 20 pacientes encaminhados para equipe de fisioterapia ao mês, estima-se a disponibilidade de quase 200 fichas de avaliação fisioterapêuticas. No entanto, levando em conta que cerca de $20 \%$ delas podem estar incompletas, estimase coletar 160 fichas para realizar esse levantamento epidemiológico.

Para a análise estatística, as variáveis dicotômicas foram apresentadas em frequência absoluta (N) e relativa (\%) e as variáveis contínuas por média e desvio padrão ou mediana e intervalo interquartil, dependendo da simetria dos dados (teste de Komolgorovic-Smirnov). Todas as análises foram realizadas com o programa Statistical Package for the Social Sciences (SPSS), na versão 17,0.

\section{RESULTADOS}

De um total de 169 prontuários, 02 deles foram excluídos por estarem incompletos. Os prontuários totalizaram 167 pacientes, com idade média 69,9 anos, predomínio $(70,7 \%)$ de idosos (>65 anos) e também do sexo feminino $(55,7 \%)$.

As doenças pulmonares foram os principais motivos de internação (35,3\%), sendo estas a pneumonia ( $N=32)$, a DPOC $(N=20)$ e o derrame pleural $(N=07)$. Sequencialmente, houve destaque para as doenças cérebro/cardiovasculares $(22,1 \%)$, tais como o AVE ( $\mathrm{N}=15)$, a insuficiência cardíaca congestiva (ICC) $(\mathrm{N}=12)$ e a HAS $(\mathrm{N}=10)$. Além disso, $71,6 \%$ dos pacientes apresentaram comorbidades e permaneceram hospitalizados por 5,0 dias (Tabela 1).

Os anti-inflamatórios não esteroides $(86,8 \%)$, seguido dos analgésicos $(49,1 \%)$, antibióticos $(46,7 \%)$ foram os fármacos mais registrados durante a hospitalização nesta amostra. Quase todos (88,0\%) os pacientes atendidos utilizaram acesso venoso periférico e apenas 15,9\% deles necessitaram de suporte nutricional (Tabela 2).

A Tabela 3 demonstra as informações do suporte ventilatório e do escore de mobilidade durante a hospitalização. Destes, 63,9\% utilizou algum dispositivo de oxigenoterapia, prevalecendo o cateter nasal $(66,3 \%)$. A aspiração de VAS foi necessária em somente 6,6\%, sendo que $34,3 \%$ eram restritos ao leito e com escore de mobilidade de 15,0 pontos.

\section{DISCUSSÃO}

A partir de nosso conhecimento, este é o primeiro estudo que investigou as características clínicas de pacientes atendidos pela equipe de fisioterapia na
Tabela 1. Características amostrais dos pacientes atendidos pela fisioterapia.

\begin{tabular}{|c|c|c|}
\hline Variáveis avaliadas $(\mathrm{N}=167)$ & $\mathbf{N}$ & $\%$ \\
\hline \multicolumn{3}{|l|}{ Características demográficas } \\
\hline Idade, anos & \multicolumn{2}{|c|}{$69,9 \pm 17,3$} \\
\hline Sexo, feminino & 93 & 55,7 \\
\hline \multicolumn{3}{|l|}{ Motivos da internação } \\
\hline Doenças pulmonares & 59 & 35,3 \\
\hline Doenças cérebro/cardiovasculares & 37 & 22,1 \\
\hline Doenças metabólicas & 22 & 13,2 \\
\hline Doenças renais & 12 & 7,2 \\
\hline Doenças neuropsicológicas & 11 & 6,6 \\
\hline Doenças gastroesofágicas & 07 & 4,2 \\
\hline Doenças oncológicas & 03 & 1,8 \\
\hline Em investigação & 16 & 9,6 \\
\hline \multicolumn{3}{|l|}{ Comorbidades ( $\mathrm{N}=155)$} \\
\hline Sim & 111 & 71,6 \\
\hline Não & 44 & 28,4 \\
\hline \multicolumn{3}{|l|}{ Tipos de comorbidades $(\mathrm{N}=155)^{*}$} \\
\hline DPOC & 63 & 40,6 \\
\hline Asma & 20 & 12,9 \\
\hline HAS & 40 & 25,8 \\
\hline ICC & 21 & 13,5 \\
\hline AVE & 25 & 16,2 \\
\hline Diabetes mellitus & 29 & 18,7 \\
\hline Obesidade & 10 & 6,4 \\
\hline Insuficiência renal crônica & 15 & 9,7 \\
\hline Pielonefrite crônica & 03 & 1,9 \\
\hline Alzheimer & 05 & 3,2 \\
\hline Parkison & 02 & 1,3 \\
\hline Epilepsia & 02 & 1,3 \\
\hline HIV & 03 & 1,9 \\
\hline Sífilis & 02 & 1,3 \\
\hline Câncer & 02 & 1,3 \\
\hline Dias de internação & \multicolumn{2}{|c|}{$5,0(2,0-9,0)$} \\
\hline
\end{tabular}

Dados expressos em frequência absoluta $(\mathrm{N})$ e frequência relativa (\%), exceto as variáveis idade (média e desvio-padrão) e dias de internação (mediana e intervalo interquartil). ${ }^{\star}$ Total do tipo de comorbidades ultrapassam as informações de 155 (100,0\%), pois há mais de uma doença associada no mesmo paciente. AVE: Acidente vascular encefálico; DPOC: Doença pulmonar obstrutiva crônica; HAS: Hipertensão arterial sistêmica; HIV: imunodeficiência humana; ICC: Insuficiencia cardíaca congestiva. 
Tabela 2. Medicações em uso (princípio ativo), acesso venoso e suporte nutricional.

\begin{tabular}{|c|c|c|}
\hline Variáveis avaliadas $(\mathrm{N}=167)$ & $\mathbf{N}$ & $\%$ \\
\hline \multicolumn{3}{|l|}{ Medicações* } \\
\hline Analgésicos & 82 & 49,1 \\
\hline Anti-inflamatórios esteroides & 75 & 44,9 \\
\hline Anti-inflamatórios não esteroides & 145 & 86,8 \\
\hline Antibióticos & 78 & 46,7 \\
\hline Betabloqueadores & 52 & 31,3 \\
\hline Bloqueadores dos canais de cálcio & 25 & 15,0 \\
\hline IECA & 46 & 27,5 \\
\hline Ansiolíticos & 33 & 19,8 \\
\hline \multicolumn{3}{|l|}{ Acesso venoso central $(\mathrm{N}=150)$} \\
\hline Sim & 07 & 4,7 \\
\hline Não & 143 & 95,3 \\
\hline \multicolumn{3}{|l|}{ Acesso venoso periférico $(N=150)$} \\
\hline Sim & 132 & 88,0 \\
\hline Não & 18 & 12,0 \\
\hline \multicolumn{3}{|l|}{ Suporte nutricional $(\mathrm{N}=145)$} \\
\hline Sim & 23 & 15,9 \\
\hline Não & 122 & 84,1 \\
\hline \multicolumn{3}{|l|}{ Tipo de suporte nutricional $(\mathrm{N}=23$ ) } \\
\hline SNE & 17 & 73,9 \\
\hline SNG & 06 & 26,1 \\
\hline
\end{tabular}

Dados expressos em frequência absoluta $(\mathrm{N})$ e frequência relativa (\%).*Total de medicação ultrapassa $167(100,0 \%)$, pois há mais de uma medicação em uso no mesmo paciente. IECA: Inibidores da enzima conversora da angiotensina; SNE: Sonda nasoenteral; SNG: Sonda nasogástrica.

enfermaria de um hospital de pequeno porte na região Litoral Norte do Rio Grande do Sul. Nossos achados evidenciaram predomínio de mulheres, da faixa etária idosa e com pouco comprometimento clínico nestes pacientes. As doenças pulmonares, como a pneumonia e a DPOC, foram as principais patologias reportadas.

A pneumonia foi o principal motivo de hospitalização, no que se refere às doenças pulmonares. Ferraz et al. ${ }^{21}$, reportaram que a pneumonia é uma morbidade altamente prevalente, sendo estimada em $58 \%$ na região Sudeste do Brasil. É considerada a terceira causa de hospitalização em idosos, gerando gastos de aproximadamente $\mathrm{R} \$ 800,00 /$ dia por prontuários no Sistema Único de Saúde (SUS) ${ }^{21,22}$. A idade avançada, o tabagismo, além das doenças crônicas (DPOC, DM e ICC) são fatores de riscos conhecidos desta enfermidade ${ }^{23}$. Isto está de acordo com as características dos pacientes deste estudo, já que grande parte deles são idosos e possuem estas comorbidades. Do mesmo modo, a exacerbação da DPOC foi à segunda causa de
Tabela 3. Suporte ventilatório e o escore de mobilidade.

\begin{tabular}{lcc}
\hline \multicolumn{1}{c}{ Variáveis avaliadas $(\mathbf{N}=167)$} & N & \\
\hline Suporte ventilatório (N=158) & 101 & 63,9 \\
Sim & 57 & 36,1 \\
Não & & \\
Tipo de suporte ventilatório (N=101) & 67 & 66,3 \\
Cateter nasal & 20 & 19,8 \\
Máscara de venturi & 14 & 13,9 \\
Máscara com reservatório & & \\
Aspiração de VAS (N=122) & 08 & 6,6 \\
Sim & 114 & 93,4 \\
Não & & 34,3 \\
Restrição ao leito (N=140) & 48 & 65,7 \\
Sim & 92 & $15,0(6,0-26,0)$ \\
Não &
\end{tabular}

Dados expressos em frequência absoluta $(\mathrm{N})$ e frequência relativa (\%), exceto a variável escala de Perme (mediana e intervalo interquartil). VAS: Vias aéreas superiores.

hospitalização no que abrange as doenças pulmonares. A agudização da doença ocorre frequentemente no inverno, sendo atribuída aos processos infeciosos que levam no aumento de mediadores inflamatórios e no incremento do volume de secreção ${ }^{24,25}$. Somando-se a isso, as doenças cérebro/cardiovasculares também se destacaram nesta amostra, obtendo predomínio do AVE e da ICC. Rossetto et al. ${ }^{4}$, corroboram com essas inferências, visto que evidenciaram a pneumonia (84\%), a DPOC (51\%) e a ICC (42\%), como as patologias mais frequentes.

Como esperado, os anti-inflamatórios, os analgésicos e os antibióticos foram os fármacos (princípios ativos) mais utilizados durante a hospitalização. De acordo com Batlouni ${ }^{26}$, os anti-inflamatórios não esteroides encontram-se entre os medicamentos mais prescritos em todo o mundo, visto que objetivam tratar a inflamação, a dor e o edema ${ }^{26,27}$. Isto é, sinais e sintomas comumente observados nos pacientes hospitalizados. Da mesma forma, 46,7\% destes indivíduos necessitaram de antibióticos. Dentre estes, podem-se listar o grupo das cefalosporinas, penicilinas e das fluorquinolonas. Esse resultado pode ser explicado devido ao uso frequente destes medicamentos para o tratamento da pneumonia e da agudização da DPOC nas afecções bacterianas ${ }^{25,28}$. Similarmente, outro estudo ${ }^{29}$ reportou o uso de antibióticos em 52,4\% da amostra em um hospital privado de Santa Maria-RS. Também está próximo dos dados divulgados por outra pesquisa ${ }^{30}$, que encontrou seu uso em $55,4 \%$ dos pacientes hospitalizados no interior de São Paulo.

No âmbito hospitalar, o uso de oxigenoterapia é esperado, uma vez que os principais motivos de 
admissão são as doenças cardiopulmonares. Dentre estes dispositivos, citam-se o cateter nasal, a máscara facial simples, a máscara de venturi e a máscara com reservatório ${ }^{31}$. Neste estudo, houve maior frequência do uso de cateter nasal, sendo este considerado um suporte ventilatório de baixo fluxo, visto que oferta até 5 litros de oxigênio por minuto ${ }^{31,32}$. Isto corrobora com um estudo anterior ${ }^{33}$, em que observou maior prevalência $(51,9 \%)$ deste dispositivo em idosos de um hospital público em Minas Gerais. Por outro lado, se contrapõe aos achados de Souza et al. ${ }^{34}$, nos quais evidenciaram maior gravidade na sua amostra, visto que os pacientes utilizavam dispositivos invasivos de oxigenoterapia, como a ventilação mecânica. Tais divergências podem ser explicadas devido as diferentes características amostrais entre os estudos e também pelos distintos setores incluídos nestas pesquisas.

Embora a escala de Perme seja comumente utilizada para estimar a mobilidade do paciente dentro da UTI, ela pode ser usada em enfermarias para o mesmo fim $^{35,36}$. Esta ferramenta avalia quinze itens, divididos em sete categorias, englobando desde o estado mental até o uso dispositivos de auxilio para deambulação e medidas de resistência ${ }^{20}$. Na presente amostra, houve um escore de 15 pontos na escala de Perme, indicando um comprometimento intermediário da mobilidade durante a hospitalização. Isto está abaixo dos resultados obtidos por outro estudo ${ }^{35}$, em que avaliou 30 pacientes submetidos a transplante hepático, obtendo uma pontuação de 31,7. Uma das possíveis explicações pode ser atribuída às diferenças entre as faixas etárias analisadas, já que o nosso estudo apresenta pacientes com idade mais avançada. Isto pode influenciar para o menor desempenho nas variáveis musculoesqueléticas avaliadas nesta escala20.

A principal limitação desta pesquisa se trata dos dados incompletos em algumas fichas de avaliação fisioterapêutica. No entanto, isto se deve ao fato de que tais informações foram registradas por inúmeros discentes do Curso de Fisioterapia. Além do mais, uma pequena parcela de pacientes encontrava-se em investigação clínica no momento da avaliação, o que se torna outra restrição do trabalho. Porém, isto se deve ao processo de investigação terapêutica, já que muitos internam por sinais/sintomas inespecíficos.

\section{CONCLUSÃO}

Os achados deste estudo evidenciaram predomínio de mulheres, da faixa etária idosa e de pouco comprometimento clínico. As doenças pulmonares, como a pneumonia e a DPOC, foram os principais motivos de hospitalização dos pacientes atendidos pela fisioterapia na enfermaria de um hospital de pequeno porte.

Com isso, espera-se auxiliar no conhecimento das características clínicas dos pacientes atendidos em um hospital desta região, visando colaborar para o direcionamento dos recursos hospitalares e de educação continuada para estes profissionais. Além do mais, estes resultados podem favorecer futuramente para a implementação de medidas de saúde pública em nível primário, considerando a importância da prevenção destas patologias na comunidade.

\section{FONTE DE FINANCIAMENTO}

Nada a declarar.

\section{CONFLITO DE INTERESSES}

Nada a declarar.

\section{REFERÊNCIAS}

1. Almeida IS, Rodrigues BMRD, Simões SMF. Hospitalização do adolescente. Rev Bras Enferm Pediatr. 2007;7(1):33-9.

2. Castro VC, Borghi AC, Mariano PP, Fernandes CAM, Mathias TAF, Carreira L. Perfil de internações hospitalares de idosos no âmbito do Sistema Único de Saúde. Rev Rede Enferm Nordeste. 2013;14(4):791-800.

3. Oliveira BRGD, Viera CS, Collet N, Lima RAGD. Causas de hospitalização no SUS de crianças de zero a quatro anos no Brasil. Rev Bras Epidemiol. 2010;13(2):268-77. http://dx.doi. org/10.1590/S1415-790X2010000200009.

4. Rossetto C, Soares JV, Brandão ML, Rosa NG, Rosset I. Causas de internação hospitalar e óbito em idosos brasileiros entre 2005 e 2015. Rev Gaúcha Enferm. 2019;40:e20190201. http://dx.doi.org/10.1590/1983-1447.2019.20190201. PMid:31644715.

5. Costa JSDD, Reis MC, Silveira CV Fo, Linhares RDS, Piccinini F, Fantinel E. Prevalência de internação hospitalar e fatores associados em Pelotas, RS. Rev Saude Publica. 2010;44(5):923-33. http://dx.doi.org/10.1590/S003489102010005000026. PMid:20802900.

6. Ferreira J, Silva JCA, Cavalcante TB, Campelo GO. Atuação do fisioterapeuta em enfermaria hospitalar no Brasil. Fisioterapia Brasil. 2017;18(6):1-8.

7. Mendes EV. 25 anos do Sistema Único de Saúde: resultados e desafios. Estud Av. 2013;27(78):27-34. http://dx.doi. org/10.1590/S0103-40142013000200003.

8. Chung TM. Escola de coluna. Acta Fisiátrica. 1996;3(2):13-7.

9. Alves AN. A importância da atuação do fisioterapeuta no ambiente hospitalar. Ensaios e Ciência. 2012;16(6):173-84.

10. Araújo CF, Tosta TB, Albuquerque DGN, Costa CC, Machado MN. A importância da fisioterapia no setor de emergência do hospital das clínicas de teresópolis costantino ottaviano. Cad Educ Saúde Fisioter. 2018;5(10):1.

11. Jerre G, Beraldo MA, Silva TDJ, Gastaldi A, Kondo C, Leme $F$, et al. Physiotherapy on the mechanically ventilated patients. Rev Bras Ter Intensiva. 2007;19(3):399-407. http://dx.doi.org/10.1590/S0103-507X2007000300023. PMid:25310080.

12. Mendes TDAB, Andreoli PBDA, Cavalheiro LV, Talerman C, Laselva C. Adequação do uso do oxigênio por meio da oximetria de pulso: um processo importante de segurança do paciente. Einstein. 2010;8(4):449-55. http://dx.doi. org/10.1590/s1679-45082010ao1377.

13. Conceição LSR, Andrade GS, Oliveira TVC, Santana LS, Andrade JD, Correia GS, et al. Perfil epidemiológico dos pacientes atendidos pela fisioterapia do Hospital da 
Universidade Federal de Sergipe. Interfaces CientíficasSaúde e Ambiente. 2014;3(1):29-38. http://dx.doi. org/10.17564/2316-3798.2014v3n1p29-38.

14. Soares JA, Silva NLS, Leite SDO, Parisi MT. Fisioterapia e qualidade de vida de paciente com amiotrofia espinal progressiva tipo I: relato de caso. Arq Ciênc Saúde. 2006;13(1):44-7.

15. Santos ACD, Biagi AC. Perfil epidemiológico dos pacientes submetidos à artroplastia de joelho do serviço de fisioterapia em hospital público de Santo André-SP. ABCS Health Sci. 2013;38(1):2-7. http://dx.doi.org/10.7322/abcshs. v38i1.2.

16. Fujisawa MAT, Valenciano PJ, Itakussu EY, Kakitsuka EE, Hoshimo AA, Trelha CS, et al. Perfil epidemiológico de crianças e adolescentes vítimas de queimaduras admitidos em centro de tratamento de queimados. Rev Bras Queimaduras. 2016;15(2):74-9.

17. Farencena GS, Silveira SN, Pasin JSM. Atuação fisioterapêutica e morbidade por pneumonia: um estudo no Hospital Casa de Saúde, Santa Maria/RS. Disciplinarum Scientia Saúde. 2006;7(1):29-39.

18. Nicolau CM, Pigo JDC, Bueno M, Falcão MC. Avaliação da dor em recém-nascidos prematuros durante a fisioterapia respiratória. Rev Bras Saúde Mater Infant. 2008;8(3):285-90. http://dx.doi.org/10.1590/S1519-38292008000300007.

19. Barata RB. Epidemiologia e políticas públicas. Rev Bras Epidemiol. 2013;16(1):3-17. http://dx.doi.org/10.1590/ S1415-790X2013000100001. PMid:23681318.

20. Kawaguchi YMF, Nawa RK, Figueiredo TB, Martins L, PiresNeto RC. Perme Intensive Care Unit Mobility Score e ICU Mobility Scale: tradução e adaptação cultural para a língua portuguesa falada no Brasil. J Bras Pneumol. 2016;42(6):42934. http://dx.doi.org/10.1590/s1806-37562015000000301. PMid:28117473.

21. Ferraz RDO, Oliveira-Friestino JK, Francisco PMSB. Tendência de mortalidade por pneumonia nas regiões brasileiras no período entre 1996 e 2012. J Bras Pneumol. 2017;43(4):2749. http://dx.doi.org/10.1590/s1806-37562016000000235. PMid:29365002.

22. Nunes SEA, Minamisava R, Vieira MADS, Itria A, Pessoa VP Jr, Andrade ALSSD, et al. Custos hospitalares de pneumonia bacteriana grave em crianças: análise comparativa por diferentes métodos de custeio. Einstein. 2017;15(2):2129. http://dx.doi.org/10.1590/s1679-45082017gs3855. PMid:28767921.

23. Gomes L. Fatores de risco e medidas profiláticas nas pneumonias adquiridas na comunidade. J Bras Pneumol. 2001;27(2):97-114. http://dx.doi.org/10.1590/S010235862001000200008.

24. Borges MC, Vianna ESO, Terra J Fo. Abordagem terapêutica na exacerbação da doença pulmonar obstrutiva crônica (DPOC). Medicina. 2003;36(2-4):241-7.

25. Fernandes FLA, Cukier A, Camelier AA, Fritscher CC, Costa $\mathrm{CH}$, Pereira EDB, et al. Recomendações para o tratamento farmacológico da DPOC: perguntas e respostas. J Bras Pneumol. 2017;43(4):290-301. http://dx.doi.org/10.1590/ s1806-37562017000000153. PMid:29365005.

26. Batlouni M. Anti-inflamatórios não esteroides: efeitos cardiovasculares, cérebro-vasculares e renais. Arq Bras Cardiol. 2010;94(4):556-63. http://dx.doi.org/10.1590/S0066782X2010000400019. PMid:20498929.

27. Guimarães DO, Momesso LS, Pupo MT. Antibióticos: importância terapêutica e perspectivas para a descoberta e desenvolvimento de novos agentes. Quim Nova. 2010;33(3):667-79. http://dx.doi.org/10.1590/S010040422010000300035.

28. Corrêa RA, Costa AN, Lundgren F, Michelin L, Figueiredo $M R$, Holanda $M$, et al. Recomendações para o manejo da pneumonia adquirida na comunidade 2018.J Bras Pneumol. 2018;44(5):405-25. PMid:30517341.

29. Rodrigues FD, Bertoldi AD. Perfil da utilização de antimicrobianos em um hospital privado. Cien Saude Colet. 2010;15(Supl 1):1239-47. http://dx.doi.org/10.1590/S141381232010000700033. PMid:20640283.

30. Fonseca LG, Conterno LO. Audit of antibiotic use in a Brazilian University Hospital. Braz J Infect Dis. 2004;8(4):27280. http://dx.doi.org/10.1590/S1413-86702004000400002. PMid:15565257.

31. Alves JCF, Fank A, Souza LP, Lima MG. O papel do enfermeiro na oxigenoterapia: uma revisão de literatura. J Health Biol Sci. 2018;6(2):176-81. http://dx.doi.org/10.12662/23173076jhbs.v6i2.1242.p176-181.2018.

32. Kock KS, Rocha PAC, Silvestre JCC, Coelho D, Leite KR. Adequações dos dispositivos de oxigenoterapia em enfermaria hospitalar avaliadas por oximetria de pulso e gasometria arterial. ASSOBRAFIR Ciência. 2014;5(1):53-64.

33. Buranello MC, Shimano SGN, Patrizzi LJ. Oxigenoterapia inalatória em idosos internados em um hospital público. Rev Bras Geriatr Gerontol. 2016;19(2):247-56. http://dx.doi. org/10.1590/1809-98232016019.140208.

34. Souza CDF, Mesquita FOS, Quinteiro MIA, Couto MCA, Correia MAVJr, Silva TNS. Perfil epidemiológico de pacientes submetidos à ventilação mecânica nas unidades de pronto atendimento de uma capital brasileira. Rev Pesq Fisioter. 2017;7(2):199-206. http://dx.doi.org/10.17267/22382704rpf.v7i2.1304.

35. Pereira CS, Carvalho AT, Bosco AD, Forgiarini LA Jr. Escala Perme como preditor de funcionalidade e complicações após a alta da unidade de terapia intensiva em pacientes submetidos a transplante hepático. Rev Bras Ter Intensiva. 2019;31(1):57-62. http://dx.doi.org/10.5935/0103507X.20190083. PMid:30970092.

36. Jerre G, Silva TDJ, Beraldo MA, Gastaldi A, Kondo C, Leme F, et al. Fisioterapia no paciente sob ventilação mecânica. J Bras Pneumol. 2007;33(Supl 2):142-50. http://dx.doi. org/10.1590/S1806-37132007000800010. 